\title{
State Anxiety Dependent on Perspiration during Mental Stress and Deep Inspiration
}

\author{
Yuri MASAOKA, Youhei ONAKA, Yuuki SHIMIZU, Shyunsuke SAKURAI, and \\ Ikuo HOMMA \\ Department of Physiology II, Showa University School of Medicine, Shinagawa-ku, Tokyo, 142-8555 Japan
}

\begin{abstract}
In this study, we focused on two types of perspiration, one triggered by anticipatory anxiety and the other by voluntary deep inspiration. We have previously found that the anticipation of anxiety causes increases of respiratory frequency, and that these increases are related to an activation of the temporal pole and amygdala in humans. Our interest is in the difference between the two natures of perspiration, both of which are closely related to respiratory responses. The level of sweating responses did not differ between deep and active inspiration or between three trials. This means that there was no habituation with repetitive trials for voluntary breathing. On the other hand, sweating responses during anticipation of anxiety showed habituation with repetitive trials. Habituation and nonhabituation differences
\end{abstract}

with respect to these two characteristics of perspiration could be the result of differences in the related central networks. The former might be involved cortical structures associated with conscious changes of respiration. The latter was induced unconsciously, and this unconscious response is similar to the respiratory response during anticipatory anxiety, which may be related to the activation of the limbic system. Further, there was a positive correlation found between the amount of perspiration response and the state anxiety scores in deep inspiration, and also in trial 1 of the anticipatory anxiety experiment. Both types of responses were related to the individual state anxiety scores, and this may contribute to the defense mechanism with regard to adjustments to changes in the outer environment and situations.

Key words: respiration, sweat gland, autonomic nervous system.

$\mathrm{O}$ ne of the essential brain functions is the regulation of the internal organs and systems to mediate the influences of the external environment. For example, body hyperthermia activates thermoregulatory responses, which are primarily controlled by the anterior hypothalamus, which is regarded as the part of the brain that handles the integration of thermal signals from the periphery [1]. The posterior hypothalamus, however, is responsible for reacting to decreases in body temperature. The sweating response is stimulated by these areas to increase the amount of heat loss. Perspiration is regulated to maintain body homeostatis. However, mental stimulation also causes perspiration, especially on the palms in humans.

Several studies have reported that mental stress or handgrip exercises can cause palmar sweating responses $[2,3]$ and also that physical tasks such as deep inspiration can mediate active palmar-/finger-sweating responses [3, 4].

We have previously found that an anticipation of anxiety causes increases of respiratory frequency, and that these increases are related to the activation of the temporal pole and amygdala in humans [5]. On the other hand, the finding that mental tasks can cause perspiration suggests that this response could be related to the activation of the amygdala and hippocampus, which have been found to have a critical role in emotion [6]. On the other hand, volitional deep inspiration causes palmar-sweating responses. Our interest is in the difference between the two natures of perspiration, both of which are closely related to respiratory responses.

In this study, we focus on the relationship between respiration and perspiration observed in anticipatory anxiety caused by mental stress and voluntary deep inspiration, and we analyze the nature of the differences in the related palmar-sweating responses.

\section{MATERIALS AND METHOD}

Subjects. Ten normal volunteers (all male; aged $30 \pm$ 3.3), who refrained from the consumption of alcohol, tobacco, or caffeine for $12 \mathrm{~h}$ prior to the experiment. All subjects had no medication at the time of the study. All subjects gave informed consent, and the study was approved by the Ethics Committee of the Showa University

Received on Jan 19, 2007; accepted on Mar 23, 2007; released online on Mar 29, 2007; doi:10.2170/physiolsci.RP000607 Correspondence should be addressed to: Ikuo Homma, Department of Physiology II, Showa University School of Medicine, 1-5-8 Hatanodai, Shinagawa-ku, Tokyo 142-8555, Japan. Phone: +81 33784 8113, Fax: +81 337840200 ,

E-mail: ihomma@med.showa-u.ac.jp 
School of Medicine. All participants were assessed with Spielberger's State Trait Anxiety Inventory (STAI) [7] to test their anxiety levels.

Measurements. All subjects were tested in two experiments in randomized order: an inspiration-induced perspiration response experiment and an emotional-induced perspiration response experiment. The subjects were measured for both respiration and perspiration during the two experiments. We measured the sweating response using a hygrometer (Kentz Perspiro OSS-100, Suzuken, Nagoya, Japan) with a probe attached to the right and the left forefingers. The ratemeter has been used for various experiments, and its reliability has been confirmed [8]. Respiratory flow and volume were measured with a flowmeter (Minato, Osaka), and perspiration responses and respiration were both stored in a computer (Sony, Japan) installed with PowerLab (AD Instruments). The parameters of the amount of perspiration, inspiration time, expiration time, total respiratory time $\left(T_{\text {tot }}\right)$, and tidal volume were analyzed by analog calculations.

\section{Protocol}

Inspiration-induced perspiration. Deep inspiration and active inspiration, specifically, inspiration of $3 / 4,1 / 2$, and $1 / 4$ of the maximum inspirable tidal volume, were tested. Participants were instructed to inspire voluntarily either deeply or actively at their own pace after taking 5 or 6 normal breaths. They were then instructed to inspire $3 / 4,1 / 2$, and $1 / 4$ of their maximum inspirable tidal volume. Fast trials were requested first to see the respiratory movements on the computer screen, and then the participants were requested to inspire for each stated volume at their own pace with an interval of 5 or 6 normal breaths.

Emotion-induced perspiration. The anticipatory experiments were performed using the same protocol as a study previously reported [9]. The subjects were informed that an electrical stimulus (ES) would be delivered to the left forefinger within two minutes after the lighting of a light- emitting diode (LED) (warning light). The time between the lighting of the LED and the ES is defined as anticipatory anxiety. The participants were notified that the ES would be painful and would cause an unpleasant sensation and that the process of the LED lighting followed by the ES would continue until the experiment was over. The participants were also informed that they could quit the study at any time.

The electrical stimulus (duration: $500 \mu \mathrm{s}$, frequency of 10 train pulse) was delivered with a stimulator (Nihon Koden, SEN-3201) and a stimulus isolator (Nihon Koden, SS-302 J). The stimulus produced a tingling sensation, and the participants reported that although it was not painful, they felt anxious after the lighting of the LED as they focused on when the ES would be delivered. All experiments were performed in a shielded room.

Statistical analysis. All statistical analyses were performed with a commercially available statistical package (SPSS, Ver.11.0; SPSS, Tokyo, Japan). Comparisons of the amount of sweating responses between deep inspiration and active inspiration were analyzed by two-way repeated measures analysis of variance (ANOVA). A Greenhouse-Geisser adjustment of the degrees of freedom was applied to the ANOVA analysis to correct for the violation of the assumption of sphericity. Comparisons of perspiration responses and total respiratory times during anticipation between trials were analyzed with a one-way repeated measures ANOVA, and a Bonferroni post hoc test was also applied.

The differences between the left and right palmar responses were also analyzed by a one-way repeated measures ANOVA. Sweating response, tidal volume, total respiratory time, and inspiratory time during rest and during each inspiratory manipulation were also analyzed by a one-way repeated measures ANOVA. Post hoc testing was performed with a Bonferroni. The correlation coefficient for the linear regression between sweating response

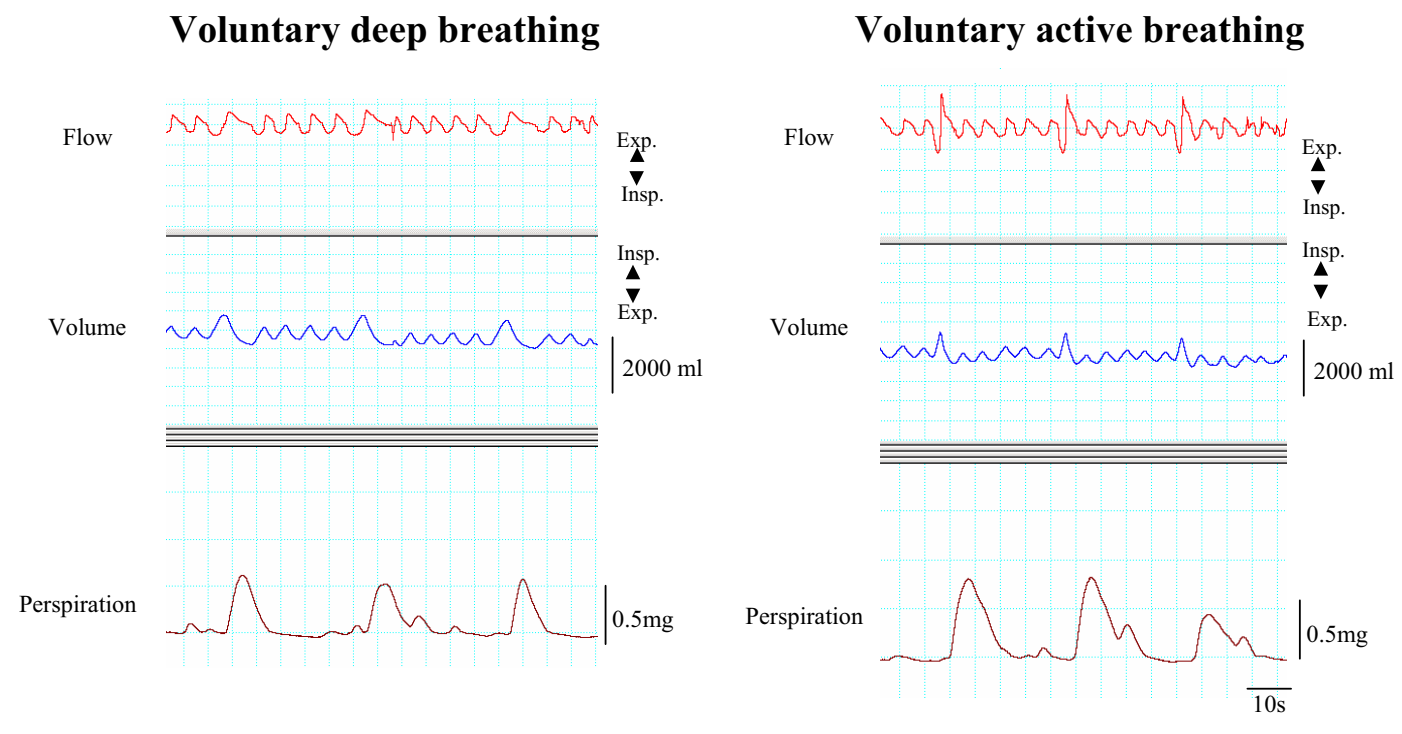

Fig. 1. Typical examples of respiratory maneuvers during voluntary deep breathing (left) and active breathing (right). Flow, volume, and perspiration corresponded with the respiratory manipulation. Flow goes downward and volume goes upward for inspiration. 
and state anxiety, and between total respiratory time and trait anxiety, were calculated. All state anxiety scores in the figures were data assessed before the experiment.

\section{RESULTS}

\section{The effects of voluntary deep breathing and active breathing on perspiration}

Figure 1 shows a typical example of sweating responses after the voluntary deep (left) and active (right) breathing, and Fig. 2 shows a typical example of respiration and sweating response during anticipatory anxiety. In Fig. 1, after the voluntary deep or active breathing, sweating re-

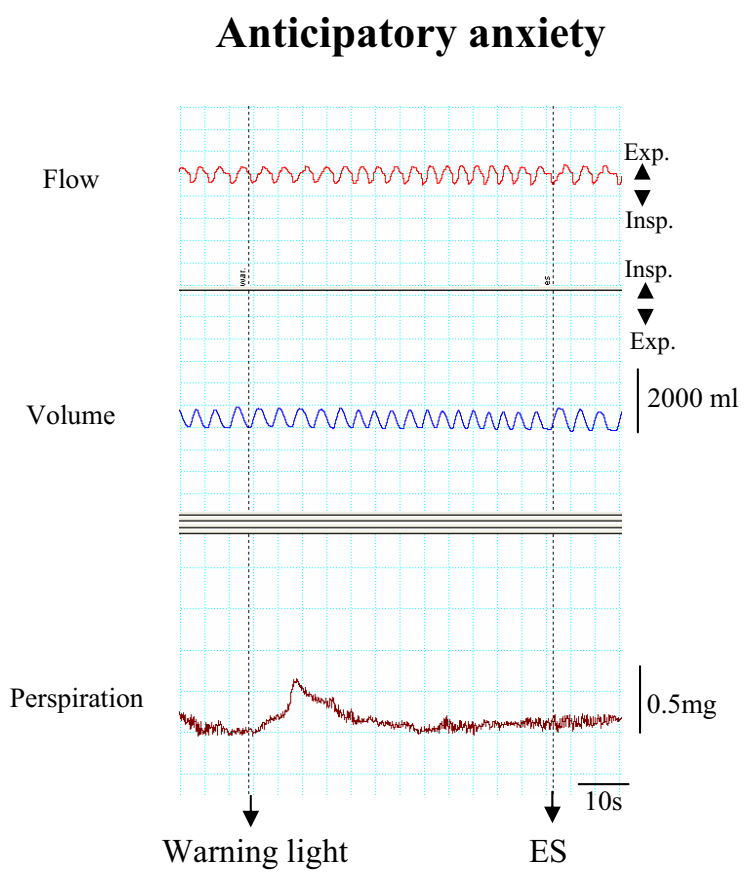

Fig. 2. Flow, volume, and perspiration during anticipatory anxiety.

Volitional inspiration

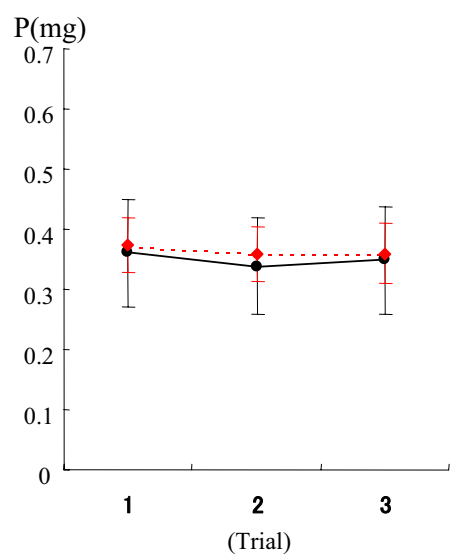

sponses were observed. These sweating response levels were not different with respect to the right- and left-hand measurements $(F=0.88, P>0.1)$, and they were not observed during voluntary deep expiration. Both voluntary deep breathing and active breathing showed sweating responses; however, the level of responses did not differ between deep and active inspiration or between three trials (Fig. 3, left, $F=0.352, P>0.5$ ). This means that there was no habituation with repetitive trials for voluntary breathing. In Fig. 2, during anticipatory anxiety (time between warning light and ES), an increase in respiratory rate and sweating response were observed. Sweating responses during the anticipation of anxiety showed habituation with repetitive trials (Fig. 3, right, $F=12.5, P<0.001$ ). There was a significant decrease in sweating response during anticipatory anxiety trials 2 and 3 , and the post hoc test showed significant decreases in trails 2 and 3 compared with trial $1(P<0.05$ respectively). Further, there was no difference between trials 2 and $3(P>0.5)$. Anticipatory anxiety decreased total respiratory time (inspiratory time + expiratory time) (Fig. 4), however, there was no habituation with repeated trials $(F=0.53, P>0.5)$.

\section{The effect of the voluntary inspiration of $3 / 4,1 / 2$, and $1 / 4$ of the maximum inspirable volume on perspiration}

Figure 5 shows the means of the perspiration, tidal volume, total respiratory time and inspiratory time during deep voluntary breathing, and voluntary inspiration of $3 /$ $4,1 / 2$, and $1 / 4$ of the maximum inspirable volume for all participants. Since there was no difference in amount of sweating response between deep and active inspiration, voluntary inspirations of $3 / 4,1 / 2$, and $1 / 4$ of the maximum deep inspiration were performed. Manipulations of the participants' breathing were confirmed by the amount of tidal volume, showing a significant difference between voluntary deep breathing $(F=18.4, P<0.001)$ and rest and other tasks (rest vs. deep voluntary breathing, deep

Anticipatory anxiety

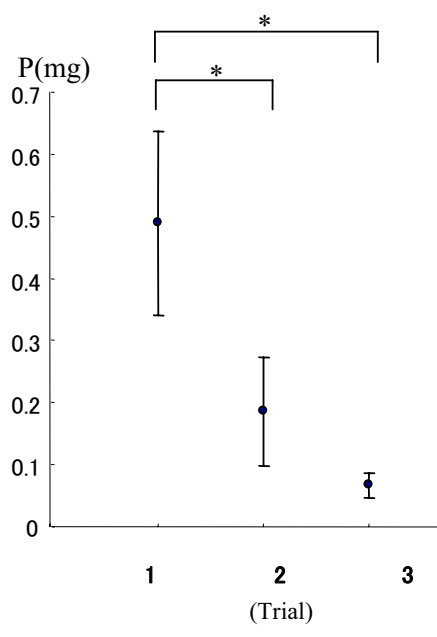

Fig. 3. Effects of repetitive deep and active inspiration (left) and anticipatory anxiety on perspiration response (right). 
voluntary breathing vs. $3 / 4,1 / 2$ and $1 / 4, P<0.001$, respectively). Total respiratory time and inspiratory time

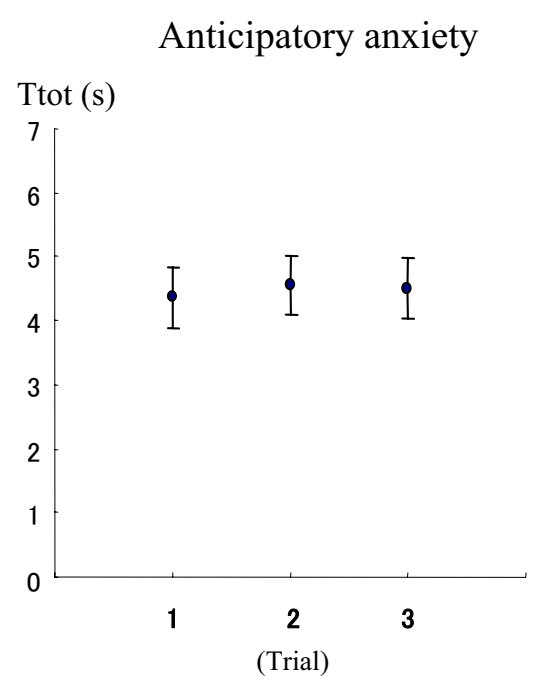

Fig. 4. Effects of repetitive anticipatory anxiety on total respiratory time $\left(T_{\text {tot }}\right)$. The data indicate all subjects' means of breaths between the lighting of the LED and the ES defined as an anticipatory anxiety.

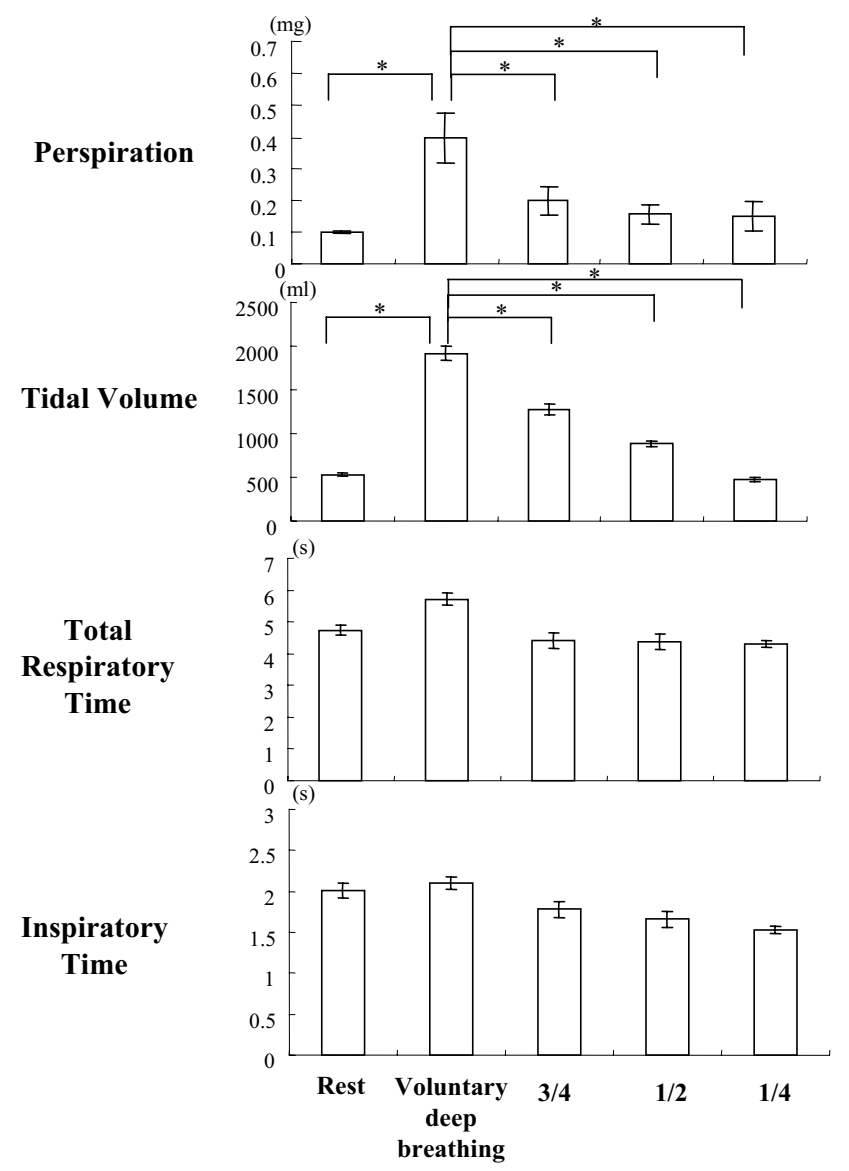

Fig. 5. Perspiration, tidal volume, total respiratory time, and inspiratory time during rest, deep voluntary breathing, and voluntary inspiration of $3 / 4,1 / 2$, and $1 / 4$ of maximum inspirable volume. ${ }^{*} P<0.001$. were the same for all trials (total respiratory time: $F=$ $3.14, P<0.001$, inspiratory time: $F=2.57, P<0.05$, however, a post hoc test showed no difference between trials, $P>0.1$, respectively). There was a significant effect of voluntary deep breathing on perspiration response $(F=$ $3.4, P<0.001$, rest vs. voluntary deep breathing, voluntary deep breathing vs. other tasks: $P<0.05$ ), and there was no difference in perspiration responses in the voluntary inspiration of $3 / 4,1 / 2$, and $1 / 4$ ( $P>0.1$, respectively).

\section{Correlation between level of sweating response and anxiety}

Figure 6 shows the correlation between sweating response during deep/active breathing and state anxiety scores. There was a positive correlation between the amount of perspiration and the state anxiety scores (deep breathing: $r=0.72, P<0.05$, active breathing: $r=0.65, P$ $<0.05$ ), however, there was no correlation between the amount of perspiration and the trait anxiety scores (deep breathing: $r=0.02, P>0.1$, active breathing: $r=0.005, P$ $>0.1$ ).

On the other hand, during anticipatory anxiety, there was a correlation between $T_{\text {tot }}$ and trait anxiety (Fig. 7), not state anxiety. Just as in previous studies $[9,10]$, there was a significant negative correlation between $T_{\text {tot }}$ and trait anxiety in anticipatory experiments $1(r=0.7, P<$ $0.05)$ and $2(r=0.5, P<0.05)$ but this correlation did not reach significance in experiment $3(r=0.3, P>0.1)$. On the other hand, the level of perspiration was correlated with the state anxiety scores in anticipatory anxiety experiment $1(r=0.7, P<0.05)$, but these correlations were suppressed in experiments $2(r=0.3, P>0.1)$ and $3(r=$ $0.1, P>0.1)$.

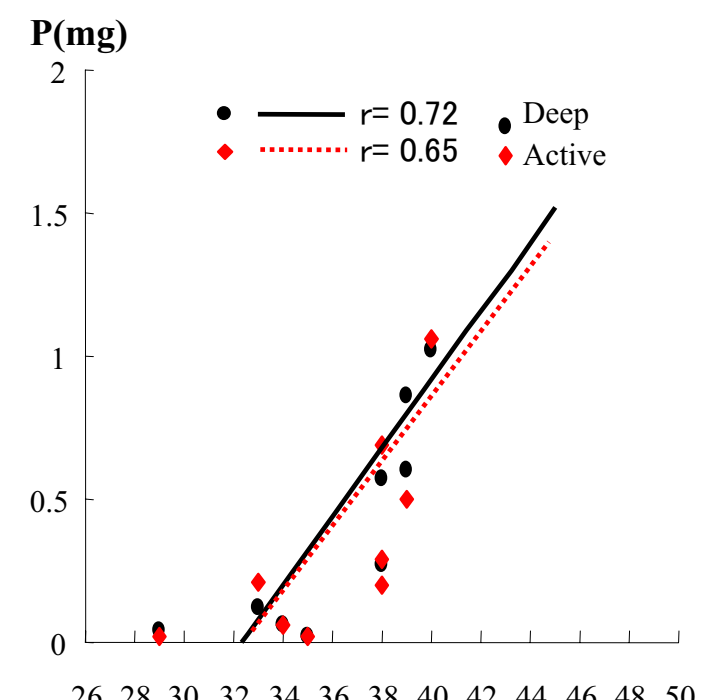

\section{State Anxiety score}

Fig. 6. Correlation between individual state anxiety scale and perspiration response. 
Anticipatory 1

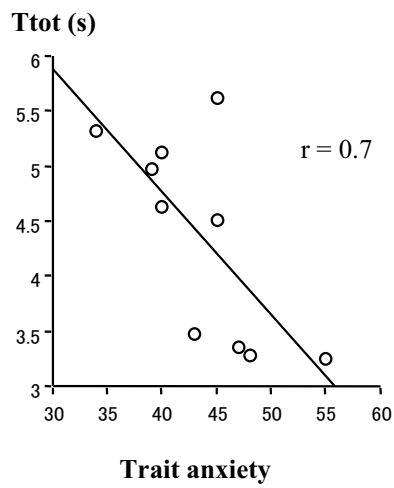

Anticipatory 1

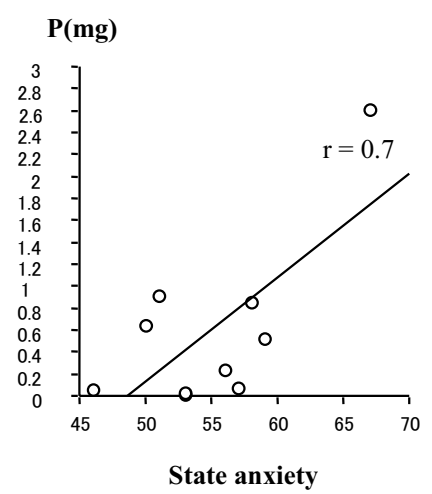

\section{DISCUSSION}

In this study, we focused on two types of perspiration, one triggered by emotional stress and the other by voluntary deep inspiration. The former was induced unconsciously, and this unconscious response is similar to the respiratory response during anticipatory anxiety, which may be related to the activation of the limbic system. The latter type, on the other hand, was intentionally induced by deep inspiration, which might involve several factors, including the vasomotor reflex and cortical structures associated with conscious changes of respiration.

\section{The effect of deep inspiration on perspiration}

The present study confirmed that voluntary inspiration (both deep and active) induced perspiration, and that the amount of perspiration was not different between deep and active inspiration. Moreover, the level of output was not dependent on the volume of the inspiration. Although sweating response was found to be related to voluntary breathing, this response occurred during deep inspiration with a large tidal volume. A study has reported that deep inspiration causes palmar sweating response, and that this response might be involved in arteriolar vasoconstriction and in transient decreases in skin blood flow [11]. The phenomenon depends on an intact peripheral sympathetic activity, and early research has concluded that the afferent stimulus caused chest wall expansion during deep inspiration. However, other research has reported that deep inspi-

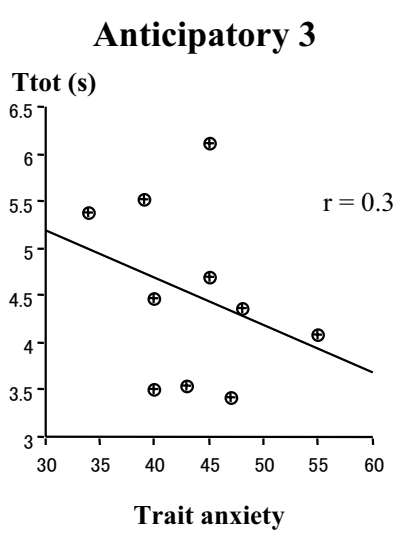

Anticipatory 3

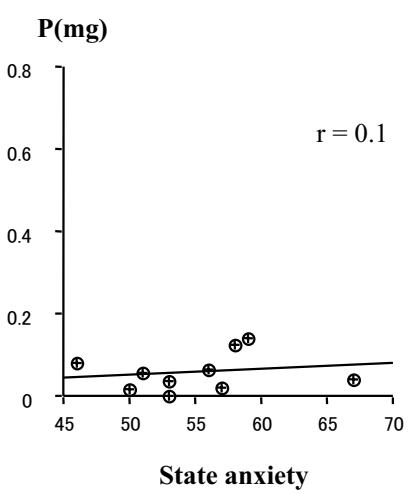

Fig. 7. Correlation between trait anxiety scale and total respiratory time $\left(T_{\text {tot }}\right)$ (upper panels) and between state anxiety scale and perspiration responses (lower panels). ration without chest wall expansion induces the same reflex, suggesting that increases in the stretch of intrathoracic veins are the principal initiating factor [12]. On the other hand, reflex vasoconstriction was not reported to be related to inspiration-induced hypotension, but rather it was associated with the carotid sinus reflex [13]. Many of these studies reported inspiration-triggered palmar-sweating responses. However, the details of the afferent and efferent pathways remain unclear.

A recent study on sudomotor nerve velocity and the central processing time of the skin conductance response (SCR) reported that inspiratory SCR onset preceded the afferent SCRs by a few hundred milliseconds [14]. This finding suggests that inspiratory SCR is unrelated to afferent involvement, but that it involves a central neural network.

We obtained no direct evidence that an activation of the higher center initiates perspiration responses in this study. However, the observed result that the intention to inspire deeply could be associated with cortical activation indicates the possible involvement of autonomic central networks.

It was of interest to find that sweating responses elicited by deep inspiration showed no habituation, but responses to the anticipation of anxiety showed adaptation. Kobayashi et al. [3] reported that active palmar sweating in response to mental stimulation was reduced one to three times with repetitive trials, but the responses to physical 
tasks were reproducible, resulting in no habituation. From these results, the conclusion can be made that palmar sweating responses in mental stress and in deep inspiration are elicited by a different mechanism, though the two systems are involved in the autonomic central networks.

\section{State anxiety and perspiration response}

There was a positive correlation found between the amount of perspiration response and the state anxiety scores in deep inspiration and also in trial 1 of the anticipatory anxiety experiment. There was a report of variability of the sweating response associated with subject-tosubject differences [3]. This variability of response could be caused by the participants' state anxiety level, which is generally measured as how one feels "right now" in a variety of situations, and it is therefore affected by various environmental and internal factors [7]. On the other hand, we consistently observed that the respiratory frequency $\left(T_{\text {tot }}\right)$ was correlated with trait anxiety during an anticipation of anxiety $[9,10]$. Trait anxiety is defined as stable individual proneness to anxiety, and its scores are generally not influenced by any conditions [7]. Previous reports suggest that increases of respiratory frequency caused by activation of the amygdala have a crucial role in fear and anxiety. For example, electrical stimulation of the amygdala was found to directly produce respiratory changes in humans [15]. Moreover, simultaneous measurement of respiration and cardiac output during anticipatory anxiety suggested that the threshold of immediate respiratory response is higher than the cardiac output because the output is regulated through the autonomic nervous system [9]. The difference between trait-dependent respiratory response and state-dependent perspiration in parallel activation of the amygdala could depend on whether the output involves the autonomic nervous system. Perspiration responses in deep inspiration and anticipatory anxiety were linked with the autonomic network, and both types of responses were related to the individual state anxiety levels, and therefore they may be influenced by various states.

\section{Habituation and nonhabituation perspiration responses}

Although the perspiration responses in deep inspiration and anticipatory anxiety were both dependent on individual state anxiety levels, the origin of each response is different; the former could be associated with a higher structure associated with motor control, and the latter relates to the limbic activation. Habituation and nonhabituation differences with respect to these two characteristics of perspiration could be the result of differences in the related central networks. In other words, the emotion-related perspiration responses could be conditioned; if the stimuli were evaluated as not harmful, the response could then be suppressed. Moreover, cortical-related perspiration involves both decision and intention, which may not include the conditioning process. However, there is direct evidence that sweating responses are not evoked in mental arithmetic or during deep inspiration in cases of damage to the bilateral amygdala [16]. The precise network involving cortical-related breathing changes and the activation of the amygdala are still unclear, but the amygdala may mediate cortical-related perspiration, and this may contribute to the defense mechanism with regard to adjustments to changes in the outer environment and situations.

\section{REFERENCES}

1. Adair ER. Skin, preoptic, and core temperatures influence behavioral thermoregulation. J Appl Physiol. 1977;42:559-64.

2. Homma S, Nakajima, Toma S, Ito T, Shibata T. Intracerebral source localization of mental process-related potentials elicited prior to mental sweating response in human. Neuroscie Lett. 1998;247:25-8.

3. Kobayashi M, Tomioka N, Ushiyama Y, Ohhashi T. Arithmetic calculation, deep inspiration or handgrip exercise-mediated pre-operational active palmar sweating responses in humans. Autonomic Neurisci Basic Clinical. 2003;104:5865.

4. Harvey N, Groseclose E.E. Inspiration-induced vascular responses in finger dorsum skin. Microvascular Res. 2002;63:227-32.

5. Masaoka Y, Homma I. The source generator of respiratory-related anxiety potential in the human brain. Neurosci Lett. 2000;283:21-4.

6. Homma S, Matsunami K, Han XY, Deguchi K. Hippocampus in relation to mental sweating response evoked by memory recall and mental calculation: a human electroencephalography study with dipole tracing. Neurosci Lett. 2001;305:1-4.

7. Spielberger CD. Manual for the State-Trait Anxiety Inventory (STAI). Palo Alto: Consulting Psychologists Press; 1983.

8. Maruyama T, Yanaga T, Makino N. Pacing-induced palmar sweating evaluated by unique hygrometer: possible implications of sympathetic activation during tachycardia. Clin Physiol. 2000;20:85-8.

9. Masaoka Y, Homma I. The effect of anticipatory anxiety on breathing and metabolism in humans. Respir Physiol. 2001;128:171-7.

10. Masaoka Y, Homma I. Expiratory time determined by individual anxiety levels in humans. J Appl Physiol. 1997;86:1329-36.

11. Bolton SA, Carmichael EA, Sturup G. Vasocontriction following deep inspiration. J Physiol. 1936;86:83-94.

12. De Lalla V. Causes of skin cooling in pressure breathing, deep inspiration and deep expiration. Am J Physiol. 1948;152:122-30.

13. Gilliatt RW. Vaso-contriction in the finger after deep inspiration. J Physiol. 1948; 107:76-88.

14. Lim CL, Seto-Poon M, Clouston PD, Morris JGL. Sudomotor nerve conduction velocity and central processing time of the skin conductance response. Clin Neurophysiol. 2003;114:2172-80.

15. Masaoka Y, Hirawasa K, Yamane F, Hori T, Homma I. Effects of left amygdala lesion on respiration, skin conductance, heart rate, anxiety and activity of the right amygdala during anticipation of negative stimulus. Behav Modif. 2003;27:607-19.

16. Asahina M, Suzuki A, Mori M, Kanesaka T, Hattori T. Emotional sweating response in a patients with bilateral amygdala damage. Int. J Psychophysiol. 2003:47:87-93. 